\title{
Anisotropy in elastic properties of lithium sodium sulphate hexahydrate single crystal-An ultrasonic study
}

\author{
GEORGE VARUGHESE*, A SANTHOSH KUMAR ${ }^{\dagger}$, J PHILIP ${ }^{\dagger \dagger}$ and GODFREY LOUIS ${ }^{\#}$ \\ Department of Physics, Catholicate College, Pathanamthitta 689 648, India \\ ${ }^{\dagger}$ School of Pure and Applied Physics, Mahatma Gandhi University, Kottayam 686 560, India \\ ${ }^{\dagger \dagger}$ Sophisticated Test and Instrumentation Centre, ${ }^{\#}$ Department of Physics, \\ Cochin University of Science and Technology, Kochi 682 022, India
}

MS received 1 October 2008; revised 22 October 2008

\begin{abstract}
The double sulfate family $\left(\mathrm{ABSO}_{4}\right)$, where $\mathrm{A}$ and $\mathrm{B}$ are alkali metal cations, is the object of great interest owing to the complexity and richness of its sequence of phase transition induced by temperature variation. A new sulfate salt characterized by the presence of water molecule in the unit cell with the chemical formula, $\mathrm{Li}_{2} \mathrm{Na}_{3}\left(\mathrm{SO}_{4}\right)_{2} \cdot 6 \mathrm{H}_{2} \mathrm{O}(\mathrm{LSSW})$, was obtained. The ultrasonic velocity measurement was done with pulse echo overlap technique [PEO]. All the six second order elastic stiffness constants, $C_{11}=C_{22}, C_{33}, C_{44}=C_{55}, C_{12}$, $C_{14}$ and $C_{13}=C_{23}$ are reported for the first time. The anisotropy in the elastic properties of the crystal are well explained by the pictorial representation of the polar plots of phase velocity, slowness, Young's modulus and linear compressibility in $a-b$ and $a-c$ planes.
\end{abstract}

Keywords. Inorganic crystal; ultrasonics; elastic properties.

\section{Introduction}

The double sulfate family $\left(\mathrm{ABSO}_{4}\right)$, where $\mathrm{A}$ and $\mathrm{B}$ are alkali metal cations, is the subject of great interest owing to the complexity and richness of its sequence of phase transition induced by either temperature or hydrostatic pressure variation. The crystals belonging to this family have similar chemical formulae, so they exhibit similar phonon spectra. The crystal, $\mathrm{Li}_{2} \mathrm{Na}_{3}\left(\mathrm{SO}_{4}\right)_{2} \cdot 6 \mathrm{H}_{2} \mathrm{O}$ (Filho et al 1999), belongs to a space group $C_{3 \mathrm{v}}{ }^{6}$ with 6 molecules per unit cell. This belongs to trigonal symmetry and the lattice parameters $a=b=8.451 \AA$ and $c=30.28 \AA$. The density of the material is measured to be $2.01518 \mathrm{~g} / \mathrm{cc}$. Raman studies of this crystal were reported by Filho et al (1999).

Hence the aim of this investigation is to measure the second order elastic constants of LSSW by ultrasonic pulse echo overlap technique and study anisotropy in the elastic properties since no report on elastic properties is available in the literature.

\section{Experimental}

\subsection{Sample preparation}

$\mathrm{Li}_{2} \mathrm{CO}_{3}$ and $\mathrm{NaHSO}_{4} \cdot \mathrm{H}_{2} \mathrm{O}$ was mixed in equi molar ratio in double distilled water. Large crystals of $\mathrm{Li}_{2} \mathrm{Na}_{3}$

\footnotetext{
*Author for correspondence (gvushakoppara@yahoo.co.in)
}

$\left(\mathrm{SO}_{4}\right)_{2} \cdot 6 \mathrm{H}_{2} \mathrm{O}$ (LSSW) were grown by slow evaporation technique at a constant temperature of $308 \mathrm{~K}$. Size of the grown single crystal, LSSW, was $(25 \times 25 \times 30) \mathrm{mm}^{3}$. The same is depicted in figure 1. During the growth of this crystal, six crystals were grown at six different temperatures from the same solution. In $323 \mathrm{~K}$ faces [002], [122], [012], [100], [010], [210], [110], [122], [212], [102] were developed. For the crystal grown at $315 \mathrm{~K}$, area of the planes [202], [022], [222] get enlarged. The morphology of certain crystals were different but the morphologies and densities of crystal grown at $313 \mathrm{~K}, 315 \mathrm{~K}$, $317 \mathrm{~K}, 319 \mathrm{~K}$ and $323 \mathrm{~K}$ were almost the same, while crystal grown at $308 \mathrm{~K}$ had entirely different morphology and different density. The new sulfate salt was characterized by the presence of water molecule in the unit cell.

The presence of water molecule was confirmed by $\mathrm{OH}$ dip at $3431 \mathrm{~cm}^{-1}$ of FTIR spectrum in figure 4. Bulk samples were cut using a slow speed diamond wheel saw so as to have propagation direction along $a$ and $c$ axes (Truell et al 1969). For trigonal crystal there were three mirror planes. Two parallel cuts were made parallel to one of the mirror planes. Three-fold symmetry planes exist in the [001] plane. A plane parallel to the mirror plane also was cut. With two axes, five of the $C_{i j}$ 's were determined. To obtain the sixth elastic constant, $C_{13}$, one had to propagate the wave along $a$-direction $45^{\circ}$ to the $z$-axis, and in a mirror plane. Faces were identified by measuring interfacial angles and comparing with the computed values. The mis-orientation was $<1^{\circ}$. The 
sample crystals along the measurement direction were in the range $0 \cdot 8-1.2 \mathrm{~cm}$. The samples were well polished by using water paper of grade 1500 and cerium oxide powder. This enables one to get proper bonding of transducer.

\subsection{Velocity measurements}

The velocity of ultrasonic waves was determined by measuring round trip time of plane ultrasonic longitudinal and transverse waves in the specimen using $X$ and $Y$-cut transducers. The ultrasonic velocities were measured using the PEO technique and details of this technique were explained elsewhere (McSkimin 1964; Papadakis 1976). A MATEC model 7700 Pulse modulator and receiver system with its associated subunits have been used for the velocity measurements.

\subsection{Elastic constant measurements of trigonal crystal}

A rhombohedral crystal has three-fold axis of symmetry and three mirror planes. It is found that $C_{11}=C_{22}, C_{33}$, $C_{44}=C_{55}, C_{12}$ and $C_{14}$ can be obtained by measuring the velocity in $c$-direction (three fold symmetry axis) and any one of the three axes in the base plane normal to a mirror plane and the third pure mode axis in the $m_{1}$ plane. The sixth elastic constant, $C_{13}=C_{23}$, can be found by velocity measurement of quasi-longitudinal wave in a mirror plane at $45^{\circ}$ with $c$-axis. The elastic constant $C_{66}$ can be obtained by knowing $C_{11}$ and $C_{12}$ by the relation $\mathrm{Li}_{2} \mathrm{Na}_{3}\left(\mathrm{SO}_{4}\right)_{2} \cdot 6 \mathrm{H}_{2} \mathrm{O}$. (LSSW) can be categorized as type- 1 trigonal with 6 independent second order elastic stiffness constants, which are $C_{11}=C_{22}, C_{33}, C_{44}=C_{55}, C_{12}, C_{13}$, $C_{14}$, and $C_{66}=1 / 2\left(C_{11}-C_{12}\right)$. The constants $C_{11}, C_{33}$ and $C_{44}$ have been determined from the direct relation, $C_{i j}=\rho V^{2}$, where $V$ is the relevant sound velocity. Eight velocity measurements enable us to determine all the seven second-order elastic constants. The ultrasonic

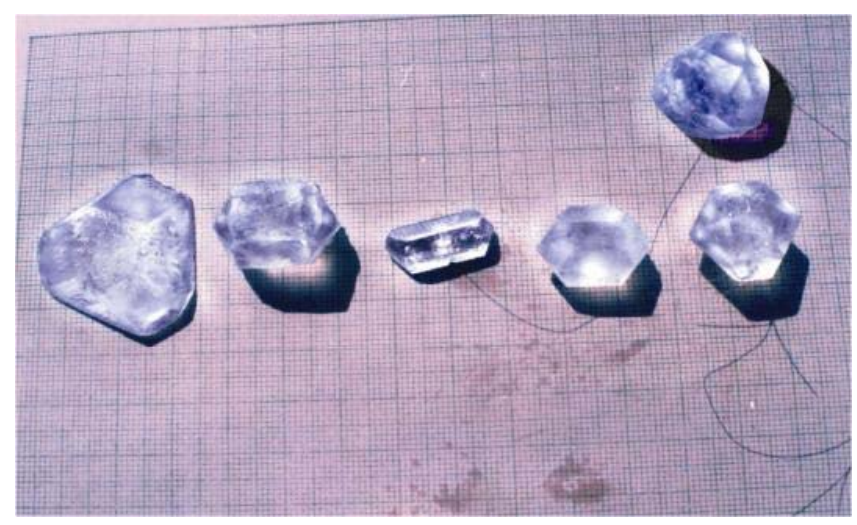

Figure 1. Photograph of lithium sodium sulphate hexahydrate $(308 \mathrm{~K})$ and lithium sodium sulphate crystals grown at $313 \mathrm{~K}$, $315 \mathrm{~K}, 317 \mathrm{~K}, 319 \mathrm{~K}$ and $323 \mathrm{~K}$. velocities in these crystals in the specified directions are tabulated in table 1 . The values of the elastic constants, compliance constants and Poisson's ratios of LSSW grown at $323 \mathrm{~K}$ are tabulated in table 2 . The elastic constants $C_{12}, C_{13}, C_{14}$ and $C_{66}$ are evaluated from the combination of other elastic constants, with the following crosschecks on the values.

$$
\begin{aligned}
& C_{11}>C_{12} ;\left(C_{11}+C_{12}\right) C_{33}>2 C_{13}^{2} ; \\
&\left(C_{11}-C_{12}\right) C_{44}>2 C_{14}^{2} .
\end{aligned}
$$

The off-diagonal constants have been derived from the Christoffel equations (Truell 1969) which are given by

$$
\begin{aligned}
& C_{14}=1 / 2\left\{\left[2 \rho v_{1}^{2}-\left(C_{66}+C_{44}\right)\right]^{2}-\left(C_{66}-C_{44}\right)^{2}\right\}^{1 / 2}, \\
& C_{12}=C_{11}+2 C_{44}-2\left(\rho v_{1}^{2}+\rho v_{2}^{2}\right), \\
& C_{13}=\frac{1}{n_{3} n_{1}}\left\{\left[\frac{C-\left(m^{3}-m^{2} A+m B\right)}{D}\right]\right\}^{\frac{1}{2}}-C_{44},
\end{aligned}
$$

where

$$
\begin{aligned}
& C_{11} n_{1}^{2}+C_{44} n_{3}^{2}=a, \quad C_{66} n_{1}^{2}+C_{44} n_{3}^{2}=b, \\
& C_{44} n_{1}^{2}+C_{33} n_{3}^{2}=c, \quad 2 C_{14} n_{1} n_{3}=l \quad \rho v_{6}^{2}=m,
\end{aligned}
$$

where

$$
\begin{aligned}
& n_{1}=1 / \sqrt{ } 2, n 3=1 / \sqrt{ } 2 \text { and } \\
& a+b+c=A, \\
& a b+b c+a c-l^{2}=B, \\
& a b c-c l^{2}=C, \\
& (a-b-2 l)=D .
\end{aligned}
$$

Here $n_{3}=\cos \theta, n_{1}=\sin \theta$, and $\theta$ is measured from $c$ axis and $\theta=45^{\circ}$. The diagonal elements can be measured with an accuracy of $0.3 \%$ and off-diagonal elastic constants to an accuracy of $1-2 \cdot 7 \%$.

\subsection{Polar plots of phase velocity, slowness, Young's modulus and linear compressibility}

The anisotropy in the propagation of elastic waves in this crystal can be illustrated clearly by plotting the phase velocity surface plot in the $x-z$ plane following the wellknown procedure (Nye 1957; Alex and Jacob 2000). The method involves solution of the Christoffel equations and numerically computing the phase velocity as a function of the direction cosines.

Figure 5 shows the phase velocity surface, in the $x-z$ plane, for the ultrasonic wave corresponding to quasi- 


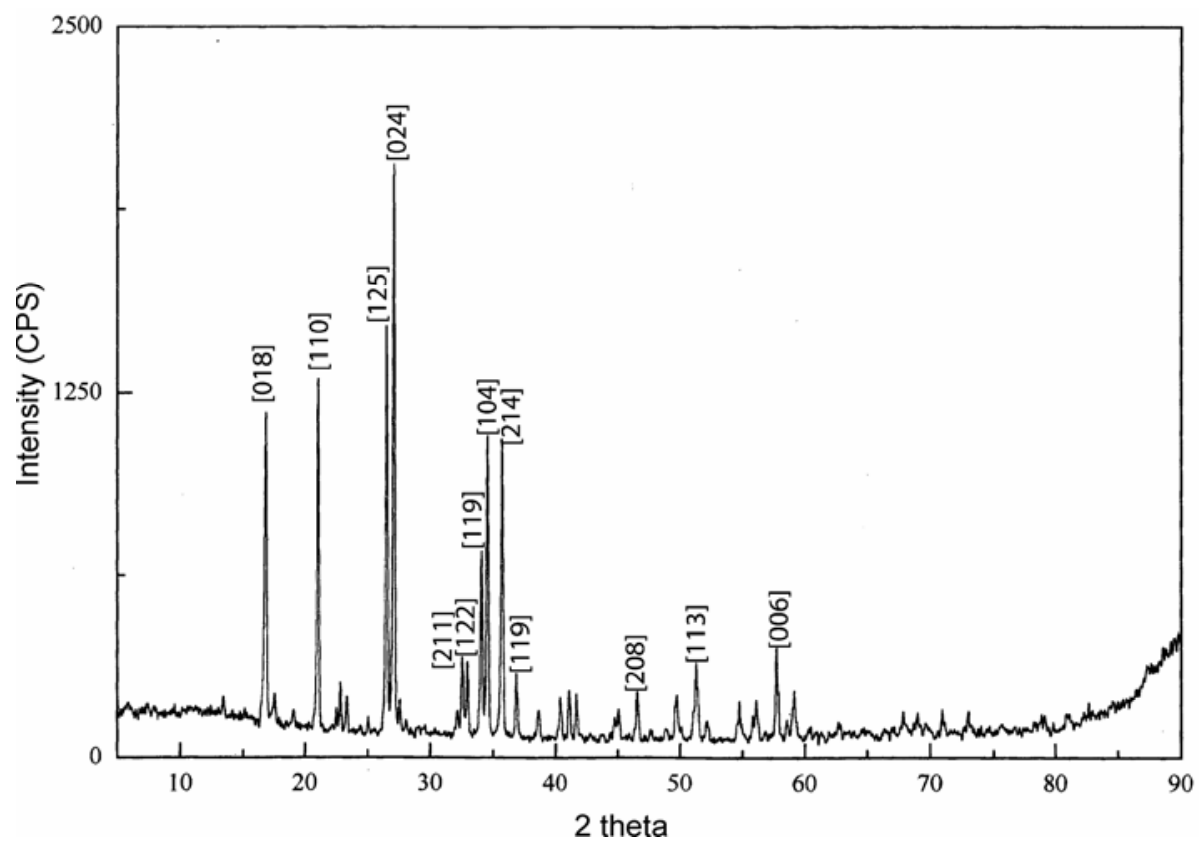

Figure 2. XRD of lithium sodium sulphate hexahydrate [LSSW] crystal.

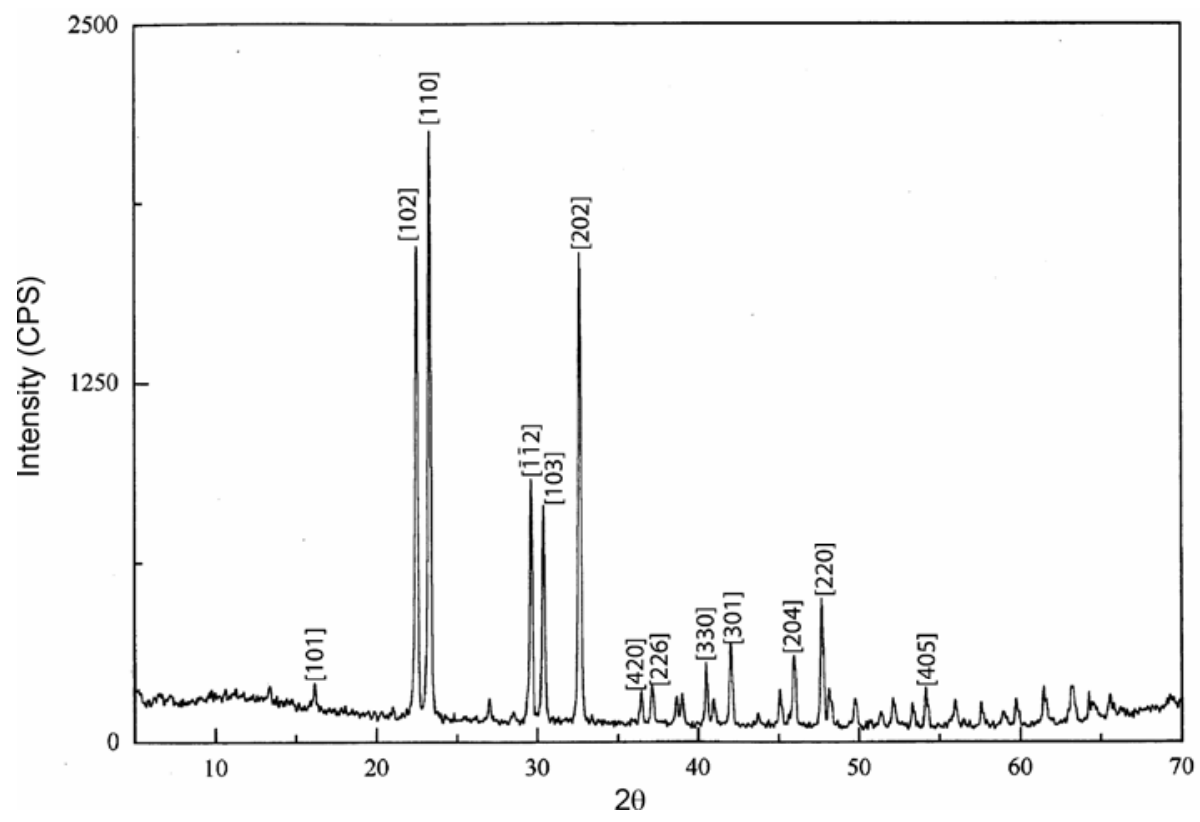

Figure 3. XRD of grown lithium sodium sulphate [LSS] crystal.

longitudinal [QT] mode. Polar plots of phase velocity and inverse of phase velocity (slowness) in other planes can also be plotted by following the same procedure and is depicted in figure 6 . The velocity surface plots outlined above alone cannot completely describe the anisotropy of the elastic properties of a crystal. The Young's moduli surface plots are also very important in this regard. The Young's modulus (Nye 1957; Alex and Jacob 2000), E, in the direction of the unit vector, $n_{i}$, for a trigonal crystal is given by

$$
\begin{aligned}
E^{-1}= & {\left[1-n_{3}^{2}\right] S_{11}+S_{33} n_{3}^{4}+\left[2 S_{12}+S_{66}\right] n_{1}^{2} n_{2}^{2} } \\
& +n_{3}^{2}\left[1-n_{3}^{2}\right]\left[2 S_{13}+S_{44}\right]+2 n_{2} n_{3}\left[3 n_{1}^{2}-n_{2}^{2}\right] S_{14} .
\end{aligned}
$$

Here $S_{i j}$ 's are the respective compliance constants.

The cross-section of Young's modulus surfaces plotted in the $x-z$ plane is shown in figure 7. The linear compressibility (Nye 1957; Alex and Jacob 2000) of a trigonal crystal in the matrix form can be written as 


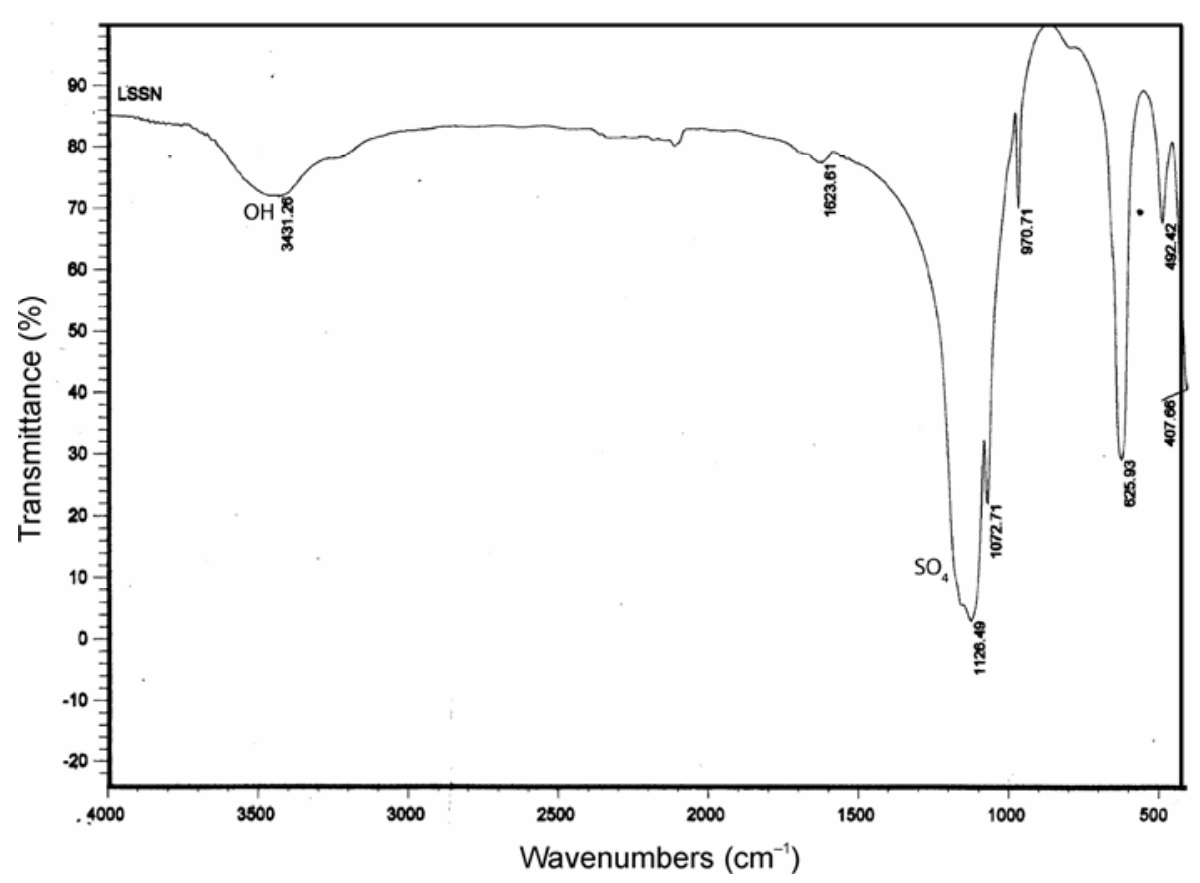

Figure 4. FTIR spectrum of lithium sodium sulphate hexahydrate crystal.

Table 1. Velocity of ultrasonic modes in LSSW at $300 \mathrm{~K}$

\begin{tabular}{lcccc}
\hline Mode & $\begin{array}{c}\text { Direction of } \\
\text { propagation }\end{array}$ & $\begin{array}{c}\text { Direction of } \\
\text { polarization }\end{array}$ & $\begin{array}{c}\text { Measured mode } \\
\text { velocity }(\mathrm{m} / \mathrm{s})\end{array}$ & $\begin{array}{c}\text { Mode velocity-elastic } \\
\text { constant relation }\end{array}$ \\
\hline $\mathrm{L}$ & {$[100]$} & {$[100]$} & $V_{1}=4769 \pm 4$ & $C_{11}=C_{22}=\rho V_{1}^{2}$ \\
$\mathrm{~T}$ & {$[100]$} & {$[001]$} & $V_{2}=2592 \pm 2$ & $C_{12}=f_{\mathrm{ab}(\mathrm{V} 2)}$ \\
$\mathrm{T}$ & {$[100]$} & {$[001] \&[010]$} & $V_{3}=2628 \pm 2$ & $C_{14}=f_{\mathrm{ab}^{\prime}}$ (V2\&V3) \\
$\mathrm{L}$ & {$[001]$} & {$[001]$} & $V_{4}=4808 \pm 4$ & $C_{33}=\rho V_{5}^{2}$ \\
$\mathrm{~T}$ & {$[001]$} & $\mathrm{QL}$ & $V_{5}=2460 \pm 2$ & $C_{44}=C_{55}=\rho V_{6}^{2}$ \\
$\mathrm{QL}$ & $\begin{array}{c}\text { Along mirror plane } \\
45^{\circ} \text { to } c \text { axis }\end{array}$ & & $V_{6}=4571 \pm 4$ & $C_{13}=f_{\mathrm{ac}(\mathrm{V} 6)}$ \\
& & & \\
\hline
\end{tabular}

L, T QL, QT represent longitudinal, transverse, quasi-longitudinal and quasi transverse modes, respectively.

$$
\beta=\left[S_{11}+S_{12}+S_{13}\right]-\left[S_{11}+S_{12}-S_{13}-S_{33}\right] n_{3}^{2} .
$$

The values of linear compressibility of LSSW crystal in the $x-y, y-z$ and $x-z$ planes have been plotted (Nye 1957; Alex and Jacob 2000). These plots are shown in figure 8 . The volume compressibility (Nye 1957; Alex and Jacob $2000), S_{i j k k}$, is an invariant parameter for a crystal. For the trigonal system, in matrix notation, it is given by

$$
S_{i i k k}=S_{33}+2\left[S_{11}+S_{12}+2 S_{13}\right]
$$

The value of the volume compressibility of LSSW is obtained as $0.22 \times 10^{-10} \mathrm{~N}^{-1} \mathrm{~m}^{2}$ and that of the bulk modulus is $45 \mathrm{GPa}$.

The Poisson's ratios (Nye 1957) of LSSW are given by the following expressions:

$$
v_{21}=-\varepsilon_{22} / \varepsilon_{11}=-S_{2211} / S_{1111}=-S_{21} / S_{11},
$$

$$
\begin{aligned}
& v_{31}=-\varepsilon_{33} / \varepsilon_{11}=-S_{3311} / S_{1111}=-S_{31} / S_{11}, \\
& v_{12}=-\varepsilon_{11} / \varepsilon_{21}=-S_{12} / S_{22}, \\
& v_{13}=-\varepsilon_{11} / \varepsilon_{33}=-S_{13} / S_{33} .
\end{aligned}
$$

These have been evaluated and are also tabulated in table 2 .

\section{Results and discussion}

A detailed study of elastic properties of the crystal has been reported for the first time. Figures 2 and 3 showed the powder X-ray diffraction pattern of lithium sodium sulphate grown at $308 \mathrm{~K}$ and $323 \mathrm{~K}$. From XRD data it was evident that structures of these two crystals are entirely different though they have been grown from the same solution. They have been compared with JCPDS 
Table 2. Elastic stiffness constant, compliance constant and Poisson's ratio of LSSW.

\begin{tabular}{llll}
\hline S1. no. & \multicolumn{1}{c}{ Elastic stiffness constant $(\mathrm{GPa})$} & $\begin{array}{c}\text { Compliance constant } \\
\left(\times 10^{-10} \mathrm{~N}^{-1} \mathrm{~m}^{2}\right)\end{array}$ & Poisson's ratio \\
\hline 1 & $C_{11}=C_{22}=45.84 \pm 0.09$ & $S_{11}=0.361$ & $v_{12}=0.169$ \\
2 & $C_{33}=46 \cdot 60 \pm 0.09$ & $S_{33}=0.393$ & $v_{21}=0.169$ \\
3 & $C_{44}=C_{55}=12.21 \times 0.02$ & $S_{44}=S_{55}=0.845$ & $v_{13}=0.417$ \\
4 & $C_{66}=15 \cdot 27 \pm 0.03$ & $S_{66}=0.845$ & \\
5 & $C_{12}=15 \cdot 3 \pm 0 \cdot 33$ & $S_{12}=-0.061$ & \\
6 & $C_{13}=2 \cdot 54 \pm 1.2$ & $S_{13}=-0.164$ & \\
7 & $C_{14}=0.648 \pm 0.19$ & $S_{14}=-0.225$ & \\
\hline
\end{tabular}

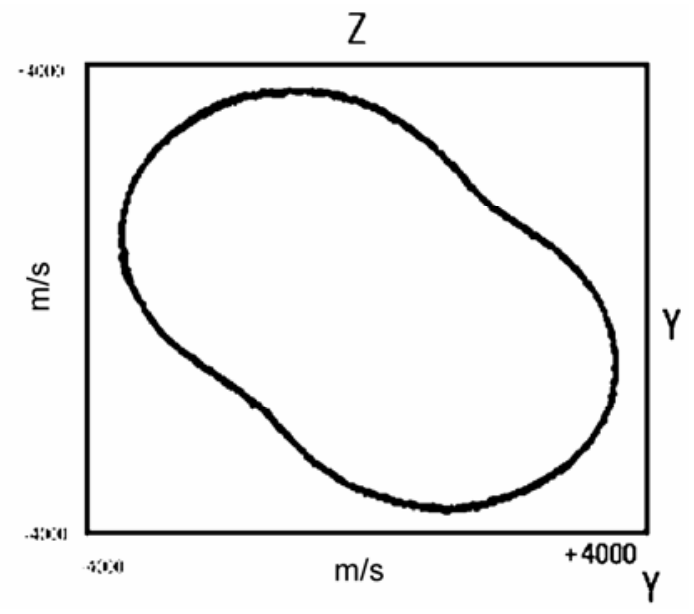

Figure 5. Polar plot of phase velocity.

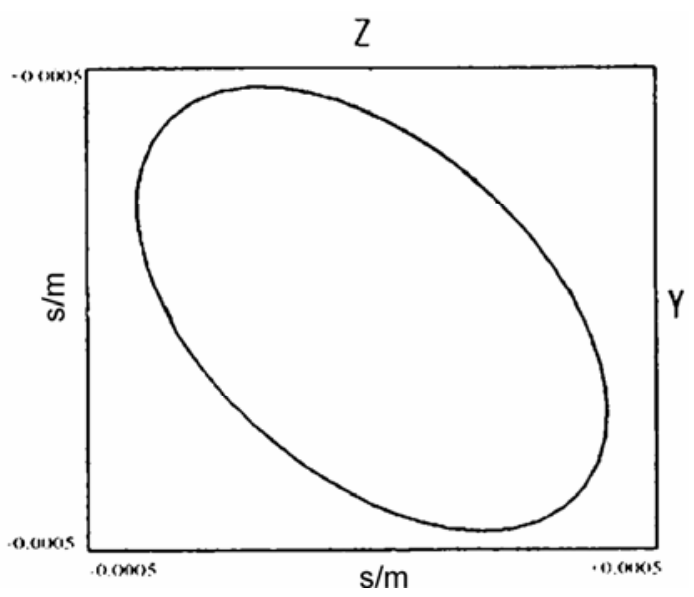

Figure 6. Polar plot of slowness

file card no. 712172 . Thus it was proved that the crystal grown at $308 \mathrm{~K}$ is not at all lithium sodium sulphate but it is $\mathrm{Li}_{2} \mathrm{Na}_{3}\left(\mathrm{SO}_{4}\right)_{2} \cdot 6 \mathrm{H}_{2} \mathrm{O}$ with different lattice parameter and space group. Some workers (Rao and Sunandana 1996; Gundusharma and Seeco 1986) have erroneously reported crystal grown at $308 \mathrm{~K}$ with ingredients $\mathrm{Li}_{2} \mathrm{CO}_{3}$ and $\mathrm{NaHSO}_{4} \cdot \mathrm{H}_{2} \mathrm{O}$ as lithium sodium sulphate.

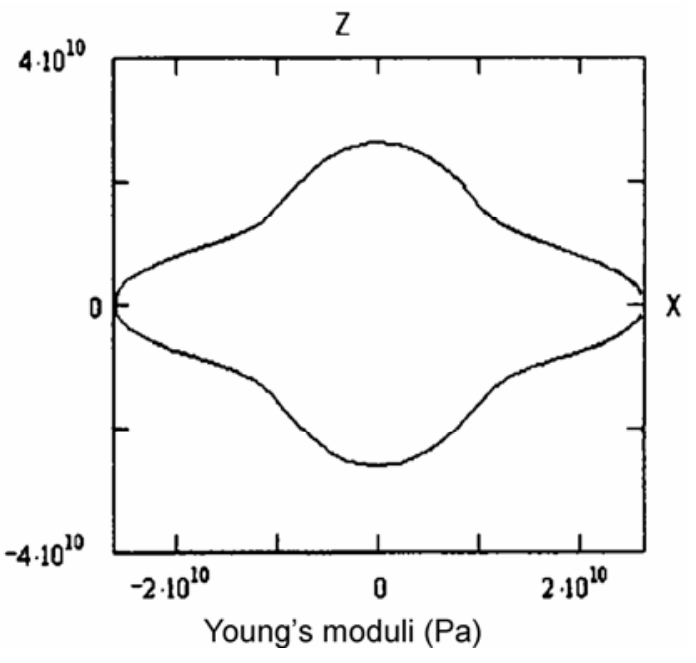

Figure 7. Polar plot of Young's modulus.

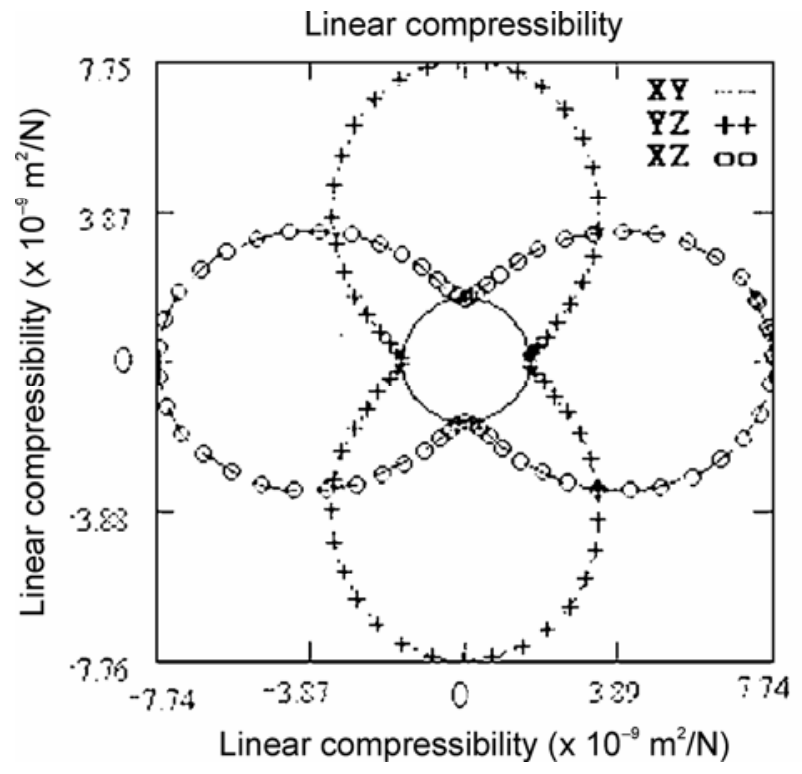

Figure 8. Polar plot of linear compressibility.

\section{Conclusions}

A detailed study of the elastic properties of LSSW gives complete set of second order elastic stiffness constants, 
compliance constants, Poisson's ratios, volume compressibility and bulk modulus of this crystal. All these important parameters for this crystal are measured and tabulated. The elastic anisotropy of various parameters like phase velocity, slowness, Young's modulus and linear compressibility are depicted in the form of twodimensional surface plots. Our present results clarified the ambiguity in the structure of this crystal grown at $308 \mathrm{~K}$.

\section{Acknowledgement}

One of the authors (GV) thanks (UGC), Delhi, for a teacher fellowship.

\section{References}

Alex A V and Jacob Philip 2000 Mater. Sci. \& Eng. B90 241 Filho J M, Paiva R O, Freire P T C and Melo F E $1999 \mathrm{~J}$. Raman Spectrosc. 30289
Gundusharma V M and Seeco E A 1986 Solid State Commun. 57479

JCPDS file card no. 712172

McSkimin H J 1964 in Physical acoustics, Part A (ed.) W P Mason (New York: Academic Press) Vol. I, p. 271

Nye N F 1957 Physical properties of crystals (London: Oxford University Press) p. 143

Papadakis E P 1976 in Physical acoustics (eds) W P Mason and R N Thurston (New York: Academic Press) Vol. XII, p. 227

Pina C M, Prieto M and Garcia L L 1995 J. Cryst. Growth 148 283

Rao S R and Sunandana C S 1996 Solid State Commun. 98 927

Truell R, Elbaum C and Chick B B 1969 in Ultrasonic methods in solid state physics (New York: Academic Press) p. 370

Walplak S and Krajewski T 1989 Phys. Status Solidi(a) 111 271 